\title{
Asymmetric Discharge-Charge Reactions in Conversion-Type Electrodes for Lithium-Ion Batteries
}

Shuang $\mathrm{Li}^{1}$, Zulipiya Shadike ${ }^{2}$, Gihan Kwon ${ }^{2}$, Ji Hoon Lee $^{3}$ and Sooyeon Hwang ${ }^{4}$

${ }^{1}$ Pacific Northwest National Laboratory, United States, ${ }^{2}$ Brookhaven National Laboratory, United States, ${ }^{3}$ Kyungpook National University, United States, ${ }^{4}$ Brookhaven National Lab, New York, United States

Conversion-type metal oxides have been actively explored as promising electrode materials for lithiumion batteries (LIBs) since much higher capacity can be achieved than well-established intercalation-type materials. However, these materials suffer from capacity fading, voltage hysteresis, and insufficient Coulombic efficiency, which limit their usage in the commercial LIBs. (Reddy et al., 2013; Cabana et al., 2010)In order to address the downside of conversion-type materials, understanding in reaction mechanisms is of importance since how reaction occurs significantly affect the fundamental electrochemical properties such as redox potential, capacity, etc. During conversion reaction (lithiation), metal oxides are completely reduced to the metallic states with the evolution of lithium oxide. (Taberna et al., 2006) In the view of microstructure, conversion reaction induces a breakdown of metal oxides into a composite of metallic nanoparticles and surrounding lithium oxides. (He et al., 2015; 2016) When reconversion occurs (delithiation), metallic nanoparticles become metal oxides. While structural changes during conversion have been explored extensively, those during reconversion is still not clear even though both reactions are evenly important for overall battery performance. Thus, elucidating the phase evolutions during delithiation can provide insights to ameliorate the electrochemical performances of conversiontype materials.

In this work, we investigate the structural changes at metal oxide during battery operations at complementary length scales. X-ray absorption spectroscopy is implemented to follow the averaged changes in the oxidation states and local bonding properties (bonding neighbors, bond length, and coordination numbers, etc.). (Jang et al., 2016; Chae et al., 2015) Also, conversion and reconversioninduced phase transformations and morphological changes are identified with transmission electron microscopy. Multimodal analyses show that conversion and reconversion occur in different reaction routes. Conversion is a direct reaction, which takes place without intermediate phases, while reconversion happens with an intermediate Li-M-O phase (M: metal). The capacity loss during electrochemical cycles is mainly originated from the unfinished delithiation reaction, evidenced by remaining Li-M-O even at the end of the delithiation stage. From the presence of the Li-M-O, we can speculate a two-step of reconversion reaction: 1) Lithium ions are extracted from $\mathrm{Li}_{2} \mathrm{O}$ and $\mathrm{M}$ migrates into the empty space in lithium oxide $\left(\mathrm{Li}_{\mathrm{x}} \mathrm{O}\right)$, resulting in the formation of $\mathrm{Li}-\mathrm{M}-\mathrm{O}$ intermediate phase. 2) Remaining Li ions are fully removed, as a result, only MO is left at the end of reconversion. This work demonstrates the phase evolutions during both lithiation and delithiation, which provides the fundamental understanding on performance decay of conversion-type materials.

References

Cabana, J., Monconduit, L., Larcher, D. \& Palacín, M. R. (2010). Beyond Intercalation-Based Li-Ion Batteries: The State of the Art and Challenges of Electrode Materials Reacting Through Conversion Reactions. Advanced Materials 22, E170-E192.

Chae, B.-M., Oh, E.-S. \& Lee, Y.-K. (2015). Conversion mechanisms of cobalt oxide anode for Li-ion battery: In situ X-ray absorption fine structure studies. Journal of Power Sources 274, 748-754. 
He, K., Xin, H. L., Zhao, K., Yu, X., Nordlund, D., Weng, T.-C., Li, J., Jiang, Y., Cadigan, C. A., Richards, R. M., Doeff, M., Yang, X.-Q., Stach, E. A., Li, J., Lin, F. \& Su, D. (2015). Transitions from Near-Surface to Interior Redox upon Lithiation in Conversion Electrode Materials. Nano Letters 15, 1437-1444.

He, K., Zhang, Sen, Li, J., Yu, X., Meng, Q., Zhu, Y., Hu, E., Sun, K., Yun, H., Yang, X.-Q., Zhu, Y., Gan, H., Mo, Y., Stach, E. A., Murray, C. B. \& Su, D. (2016). Visualizing non-equilibrium lithiation of spinel oxide via in situ transmission electron microscopy. Nature Communications 7, 11441.

Jang, J.-H., Chae, B.-M., Oh, H.-J. \& Lee, Y.-K. (2016). Understanding conversion mechanism of NiO anodic materials for Li-ion battery using in situ X-ray absorption near edge structure spectroscopy. Journal of Power Sources 304, 189-195.

Reddy, M. V., Subba Rao, G. V. \& Chowdari, B. V. R. (2013). Metal Oxides and Oxysalts as Anode Materials for Li Ion Batteries. Chemical Reviews 113, 5364-5457.

Taberna, P. L., Mitra, S., Poizot, P., Simon, P. \& Tarascon, J.-M. (2006). High rate capabilities Fe304-based Cu nano-architectured electrodes for lithium-ion battery applications. Nature Materials 5, 567-573. 